\title{
Validating two questions in the Force Concept Inventory with subquestions
}

\author{
Jun-ichiro Yasuda* \\ Center for General Education, Gifu University, Gifu, Yanagido 1-1, Japan \\ Masa-aki Taniguchi \\ Comprehensive Scientific Education Center, Meijo University, Tempaku, Nagoya, Japan \\ (Received 26 November 2012; published 5 April 2013)
}

\begin{abstract}
In this study, we evaluate the structural validity of Q.16 and Q.7 in the Force Concept Inventory (FCI). We address whether respondents who answer Q.16 and Q.7 correctly actually have an understanding of the concepts of physics tested in the questions. To examine respondents' levels of understanding, we use subquestions that test them on concepts believed to be required to answer the actual FCI questions. Our sample size comprises 111 respondents; we derive false-positive ratios for prelearners and postlearners and then statistically test the difference between them. We find a difference at the 0.05 significance level for both Q.16 and Q.7, implying that it is possible for postlearners to answer both questions without an understanding of the concepts of physics tested in the questions; therefore, the structures of Q.16 and Q.7 are invalid. In this study, we only evaluate the validity of these two FCI questions; we do not assess the validity of previous studies that have compared total FCI scores.
\end{abstract}

DOI: 10.1103/PhysRevSTPER.9.010113

PACS numbers: 01.40.Fk

\section{INTRODUCTION}

Numerous types of diagnostic tools have been studied to examine how students learn about physics. One such diagnostic tool, the Force Concept Inventory (FCI) developed by Hestenes, Wells, and Swackhamer, is a renowned instrument for assessing students' understanding of the Newtonian conceptual framework [1-4]. The FCI, which has been translated into over 24 languages and is internationally used in the field of physics education, is a 30-item, five-choice survey that can be solved almost entirely without the use of equations. Further, the distractors in the questions are constructed based on naïve conceptions about mechanics.

When conducting a survey using a diagnostic tool such as the FCI, it is first necessary to analyze its validity [5]. Validity refers to whether the instrument actually measures the construct it is supposed to [6]. In the case of the FCI, therefore, we must investigate whether the FCI accurately assesses students' conceptual learning of Newtonian dynamics.

The FCI has previously been validated from various standpoints. Hestenes and colleagues evaluated the validity of the wording and diagrams in its questions [1,7], while Rebello and Zollman analyzed the validity of the distractors in the questions by comparing students' responses to

\footnotetext{
*ysdjun16@gifu-u.ac.jp
}

Published by the American Physical Society under the terms of the Creative Commons Attribution 3.0 License. Further distribution of this work must maintain attribution to the author(s) and the published article's title, journal citation, and DOI. four FCI open-ended questions [8]. Morris and colleagues also evaluated the validity of the distractors by analyzing the item response curves $[9,10]$, and Stewart and colleagues validated the contexts of the questions using a ten-question context-modified test [11].

In this study, we focus on the validity of the FCI's structure. Put simply, our research question asks whether students who respond correctly to an FCI question actually understand the physics concept that question is meant to test. In a previous study, Yasuda and colleagues used a semistructured interview to evaluate the Japanese translation of the FCI and found structural inadequacies in Q.16 (Fig. 1) [12]. Students were able to provide the correct answer to Q.16 even when using the incorrect reasoning that the forces were balanced because the two vehicles were moving at a constant speed. Similar shortcomings have been highlighted by other studies. For example, Thornton and colleagues used the interview method to highlight the inadequacy of Q.37 in force and motion concept evaluation [13], and Scott and colleagues performed a factor analysis on an FCI data set and hypothesized why students who answer Q.16 correctly treat it as a question pertaining to Newton's first law rather than to his third law [14].

However, previous studies are not sufficient to examine the significance of this acknowledged inadequacy in the structure of Q.16. Yasuda and colleagues found that eight out of ten students who answered Q.16 correctly provided the wrong reasoning for their answers [12]. However, this finding was not considered statistically significant because the number of respondents was insufficient. Scott and colleagues also stated that their hypothesis requires further investigation [14]. 


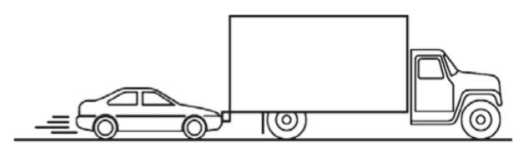

A large truck breaks down out on the road and receives a push back into town by a small compact car as shown in the figure above. After the car reaches the constant cruising speed at which its driver wishes to push the truck:

1. The amount of force with which the car pushes on the truck is equal to that with which the truck pushes back on the car.

2. The amount of force with which the car pushes on the truck is smaller than that with which the truck pushes back on the car.

3. The amount of force with which the car pushes on the truck is greater than that with which the truck pushes back on the car.

4. The car's engine is running so the car pushes against the truck, but the truck's engine is not running so the truck cannot push back against the car. The truck is pushed forward simply because it is in the way of the car.

5. Neither the car nor the truck exert any force on the other. The truck is pushed forward simply because it is in the way of the car.

FIG. 1 (color online). Q.16 of the FCI [15].

Based on the foregoing, the present paper serves to bridge this gap in the literature. Specifically, we develop a survey method to effectively increase the number of respondents in order to statistically analyze the inadequacy of Q.16. We use questions (termed subquestions herein) that elicit information on the concepts required to answer this FCI question [16]. Using subquestions, we survey two FCI questions (Q.16 and Q.7) and statistically analyze the significance of the inadequacy of these questions.

The remainder of this paper is organized as follows. Section II contains the data collection procedure and the analyzed data. In Sec. III, we describe the results of our survey, and in Sec. IV, we discuss the validity and limitations. Section V offers a summary of this study.

\section{PROCEDURE}

\section{A. Data collection}

We surveyed 111 students at Meijo University from January to April 2012. We excluded from the analysis the responses of students who did not answer some of the questions. Therefore, the number of valid responses was 108 and 107 for Q.16 and Q.7, respectively. Respondents comprised students from different departments (e.g., engineering, agriculture, human studies [17]), and most were students in the university's physics classes (e.g., calculusbased mechanics, general physics). Since the survey was conducted during the lecture time associated with the class activity, students were able to concentrate on the survey.
They were not given any incentive to participate (in the form of money or grade points). By way of ethical considerations, we explained to students the objectives of the study, the extent to which the results would be disclosed, and how their personal data would be treated. We then confirmed their agreement to these conditions before beginning the survey.

\section{B. Method of analysis}

First, we evaluated the false positives in the responses in order to assess the structural validity of the two investigated FCI questions [7]. False positives refer to correct answers provided by students who actually do not understand the physics concept being tested in the questions (see Table I). The FCI has five multiple-choice answers per question; therefore, if a respondent chooses the correct answer randomly, false positives will appear in $20 \%$ of his or her answers. Thus, we focused on highly frequent false positives to validate the question structures. False negatives, by contrast, refer to incorrect answers provided by students who actually understand the physics concept tested in the question (see Table I). Although false negatives include careless errors, we must still consider them carefully in the validation because respondents are responsible for such mistakes.

Next, it was necessary to examine whether respondents understood the concepts tested in the questions in order to evaluate the false positives. In our previous study [12], although we used interviews to make our assessment, we faced the problem of inconsistency because judgment ultimately depends on the interviewer. Therefore, in this survey, we used the system of subquestions. If a respondent answered all subquestions correctly, we assumed that he or she had an understanding of the physics concept tested. The decision table of answers with subquestions is presented in Table II.

TABLE I. Decision table of answers.

\begin{tabular}{lccc}
\hline \hline & \multicolumn{2}{c}{ Real state of a respondent } \\
& & Understands & Does not understand \\
\hline \multirow{2}{*}{ Result } & Correct & True positive & False positive \\
& Incorrect & False negative & True negative \\
\hline \hline
\end{tabular}

TABLE II. Decision table of answers with subquestions. ${ }^{\text {a }}$

\begin{tabular}{ccc}
\hline \hline & \multicolumn{2}{c}{ Subquestions } \\
& All correct answers (B) & $\begin{array}{c}\text { Not all correct } \\
\text { answers (Not B) }\end{array}$ \\
\hline \multirow{4}{*}{$\begin{array}{c}\text { Correct } \\
\text { Question }\end{array}$} & True positive & False positive \\
Incorrect & (A and B) & (A and not B) \\
(Not A) & (Not A and B) & True negative \\
(Not A and not B)
\end{tabular}

${ }^{\mathrm{a}}$ Each item corresponds to items in Table I. 


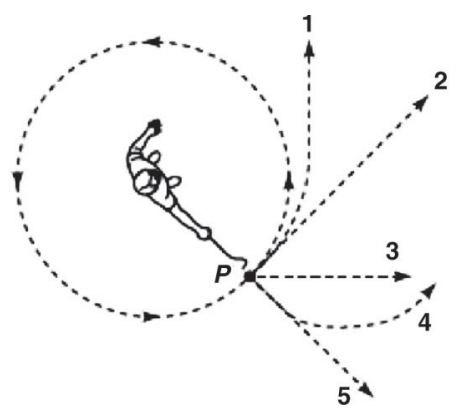

A steel ball is attached to a string and is swung in a circular path in a horizontal plane as illustrated in the accompanying figure. At the point $\mathrm{P}$ indicated in the figure, the string suddenly breaks near the ball. If these events are observed from directly above as in the figure, which path would the ball most closely follow after the string breaks?

FIG. 2 (color online). Q.7 of the FCI.

We focused on Q.16, in which we found a substantial number of false positives, and Q.7 (Fig. 2), in which we found a few, but not a negligible number of false positives. The physics concept tested in Q.16 was the Newton's third law, while that in Q.7 was the uniform circular motion [18]; therefore, we designed the subquestions of Q.16 and Q.7 as shown in Figs. 3 and 4, respectively [19]. The drawback of Q.16 is that students can arrive at the correct answer through the incorrect reasoning that the forces are balanced because both vehicles are moving at a constant speed. Therefore, our subquestions were designed to check whether students were confusing Newton's first law with Newton's third law. Q.7 also has the problem wherein students could provide the correct answer with the wrong

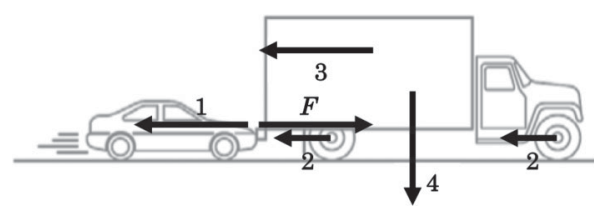

In the same situation as Q.16 (the car is moving at a constant speed):

SQ.1 Which of the forces 1-4 above best represents the force that balances with the force with which the car pushes on the truck?

SQ.2 Which of the forces $1-4$ above best represents the reaction force to the force with which the car pushes on the truck?

1. Force with which the truck pushes on the car

2. Frictional force acting on the truck

3. Inertial force with which the truck is going to remain in the same place

4. Gravitational force acting on the truck

FIG. 3 (color online). Subquestions of FCI Q.16.
SQ.1 For the observer on the ground, which of the seven choices best represents the direction of the force acting on the ball just after the string breaks?

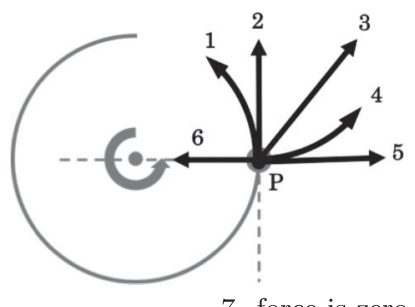

SQ.2 For the observer on the ground, which of the seven choices best represents the direction of the acceleration of the ball just after the string breaks?

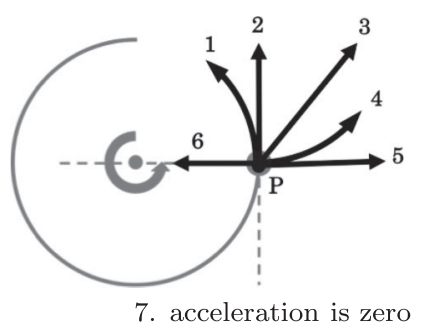

SQ.3 For the observer on the ground, which of the seven choices best represents the direction of the velocity of the ball just after the string breaks?

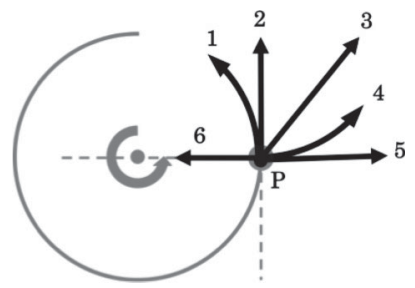

7. velocity is zero

FIG. 4 (color online). Subquestions of FCI Q.7.

reasoning that the direction of the acceleration (or the direction of the force acting on the ball) is the same as the direction of the velocity after the string breaks. Therefore, our subquestions aimed to ascertain students' understanding of the directions of velocity, acceleration, and the force acting on the ball after the string breaks. It was important to ensure that the subquestions did not inadvertently hint at the answer to the actual FCI questions; therefore, the subquestions were only asked after the students responded to the FCI questions; moreover, students were not allowed to return to previous questions.

We analyzed the false-positive results by evaluating the false-positive ratio. If event $\mathrm{A}$ represents answering a question correctly and event B represents answering all the related subquestions correctly (see Table II), then the false-positive ratio of that question is defined as follows:

$$
P(\mathrm{~A} \mid \operatorname{not} \mathrm{B})=\frac{N(\mathrm{~A} \text { and not } \mathrm{B})}{N(\operatorname{not} \mathrm{B})},
$$

where $N$ (A and not $\mathrm{B}$ ) is the number of students who answered an FCI question correctly (Q.16 or Q.7) and answered more than one of the subquestions incorrectly, and $N$ (not B) refers to the number of students who answered more than one of the subquestions incorrectly. In this case, evaluating the false-positive ratio can be interpreted as identifying the subgroup that does not 
understand the physics concept tested in the question and calculating the percentage of correctly answered questions.

In the next step, we examined the validity of the question's structure by comparing the false-positive ratios of students who had and had not studied the physics concept tested. From the questionnaire responses, we determined whether students had studied the Newton's third law or uniform circular motion. If students answered yes to having studied either concept, we called them postlearners and prelearners otherwise.

We called the false-positive ratios of prelearners $P_{1}$ and those of postlearners $P_{2}$. As the structure of the question is valid, it follows that only if students cannot understand the physics concept will they answer incorrectly, except in cases of coincidence. Therefore, if we choose the subgroups that do not understand the physics concept tested from both prelearners and postlearners, the percentage of questions answered correctly for each subgroup should be comparable. However, there is one case in which $P_{1}$ and $P_{2}$ are not comparable: when the postlearner responds correctly by using an incorrect physics concept or by remembering the correct answer of a similar question. In this case, the false-positive ratio of postlearners could become large. Therefore, if $P_{2}$ is significantly larger than $P_{1}$, we examine whether the question is not valid because postlearners can correctly answer the question even if they have no understanding of the physics concept tested.

We thus used a statistical hypothesis test to assess the significant difference between $P_{1}$ and $P_{2}$. The procedure for testing significance was as follows. First, we used the null hypothesis that there is no significant difference between the populations of $P_{1}$ and $P_{2}$ together. The standardized quantity, $Z$ score, was given as

$$
Z_{12}=\frac{P_{1}-P_{2}}{\sqrt{\hat{P}_{12}\left(1-\hat{P}_{12}\right)\left(1 / N_{1}+1 / N_{2}\right)}},
$$

where $N_{1}$ and $N_{2}$ refer to the prelearners and postlearners, respectively, who incorrectly answered more than one subquestion; $\hat{P}_{12}$ is then defined as follows:

$$
\hat{P}_{12}=\frac{N_{1} P_{1}+N_{2} P_{2}}{N_{1}+N_{2}} \text {. }
$$

When $N_{1}$ and $N_{2}$ are large enough $(N \geqslant 30)$, the $Z$ score follows a standard normal distribution. We then determined whether the null hypothesis was rejected by comparing the $Z$ score with the significant point $Z_{0}$ at a certain significance level. For example, at the 0.05 significance level [20], $\left|Z_{0}\right|$ is 1.96 . If $|Z|$ is larger than $\left|Z_{0}\right|$, the abovementioned null hypothesis can be said to be rejected and we determined that there was a significant difference between $P_{1}$ and $P_{2}$ at the 0.05 significance level. On the contrary, if $|Z|$ is smaller than $\left|Z_{0}\right|$, we determined there to be no significant difference between $P_{1}$ and $P_{2}$ at the 0.05 significance level.

\section{RESULTS}

The results of our survey are presented in Table III. The false-positive ratios for Q.16 were $P_{1}=0.26$ and $P_{2}=0.50$. The number of prelearners and postlearners who responded incorrectly to the subquestions were $N_{1}=31$ and $N_{2}=48$, respectively. Using these values, we calculated the $Z$ score from Eqs. (2) and (3) and obtained $Z_{12}=2.14$. This value was larger than $\left|Z_{0}\right|=1.96$, which was the point of significance at the level of 0.05 . Therefore, with regard to Q.16, we determined there to be a significant difference between $P_{1}$ and $P_{2}$.

Similarly, the false-positive ratios for Q.7 were $P_{1}=0.39$ and $P_{2}=0.71$. The number of prelearners and postlearners who responded incorrectly to the subquestions were $N_{1}=46$ and $N_{2}=48$, respectively. Using these values, we obtained a $Z$ score of 3.09. This value was larger than $\left|Z_{0}\right|=1.96$. Therefore, with regard to Q.7, we ascertained there to be a significant difference between $P_{1}$ and $P_{2}$.

Based on these results, we can state that it is possible for postlearners to answer both questions without an understanding of the physics concepts tested in the questions; therefore, the structures of Q.16 and Q.7 are invalid.

We also compared the false-positive ratios with the results of the interview from our previous study. These are also presented in Table III. During the interview, if the respondent was able to provide the right rationale for a correctly answered question, we assessed whether he or

\begin{tabular}{|c|c|c|c|c|c|c|}
\hline & \multicolumn{3}{|c|}{ FCI Q.16 } & \multicolumn{3}{|c|}{ FCI Q.7 } \\
\hline & Prelearners & Postlearners & $\begin{array}{c}\text { Postlearners } \\
\text { (Interview) }\end{array}$ & Prelearners & Postlearners & $\begin{array}{c}\text { Postlearners } \\
\text { (Interview) }\end{array}$ \\
\hline Number of false positives: $N(\mathrm{~A}$ and not $\mathrm{B})$ & 8 & 24 & 8 & 18 & 34 & 3 \\
\hline Number of incorrect students: $N($ not B $)$ & $N_{1}=31$ & $N_{2}=48$ & $N_{3}=13$ & $N_{1}=46$ & $N_{2}=48$ & $N_{3}=5$ \\
\hline False-positive ratio: $P(\mathrm{~A} \mid \operatorname{not} \mathrm{B})$ & $P_{1}=0.26$ & $P_{2}=0.50$ & $P_{3}=0.62$ & $P_{1}=0.39$ & $P_{2}=0.71$ & $P_{3}=0.60$ \\
\hline $\begin{array}{l}Z \text { score of the difference between } \\
P_{2} \text { and } P_{n}: Z_{2 n}\end{array}$ & $Z_{12}=2.14^{*}$ & & $Z_{23}=-0.74$ & $Z_{12}=3.09 *$ & & $Z_{23}=0.50$ \\
\hline
\end{tabular}

TABLE III. False-positive ratios and $Z$ scores for FCI Q.16 and Q.7. ${ }^{\text {a }}$

${ }^{a}$ Each numerical value is indicated up to two decimal places. One star (*) represents there was a significant difference between $P_{2}$ and each item at the 0.05 level. The results for the postlearners in the interview are reference values since the sample size was too small. 
she had an understanding of the physics concept tested in the question. In this case, the false-positive ratio of Q.16 for postlearners was $P_{3}=0.62$, and the number of students who could not provide the correct reasoning was $N_{3}=13$. With these values and the same analysis as $P_{1}$ and $P_{2}$, we obtained the $Z$ score of the difference between $P_{2}$ and $P_{3}$ : $Z_{23}=-0.74$. The absolute value of $Z_{23}$ was smaller than $\left|Z_{0}\right|=1.96$. Therefore, with regard to Q.16, we determined there to be no significant difference between $P_{2}$ and $P_{3}$. Similarly, the false-positive ratio of Q.7 for postlearners was $P_{3}=0.60$, and the number of students who could not provide the correct reasoning was $N_{3}=5$. We obtained $Z_{23}=-0.50$, and the absolute value of $Z_{23}$ was smaller than $\left|Z_{0}\right|=1.96$. Therefore, with regard to Q.7, we also determined there to be no significant difference between $P_{2}$ and $P_{3}$.

The above arguments point to a consistency in the findings between this survey and the interviews conducted as a part of the previous study. However, as mentioned earlier, the drawback of the interview method is that the final judgment on whether respondents understand the physics concept being tested depends on the interviewer, which may lead to inconsistencies. Moreover, the sample size used in the previous study was too small. Therefore, a comparison between the results of this survey and those from the interview can only be used as reference material.

\section{DISCUSSION}

\section{A. Validity of the subquestions}

We evaluated the validity of the subquestions from two perspectives: (1) the number of subquestions and (2) the contents of the subquestions. To evaluate validity, we analyzed the false positives for the subquestions of Q.7. We defined event $\mathrm{C}$ as answering some subquestions correctly in order to evaluate the false-positive ratios for each case, as shown in Table IV.

\section{Number of subquestions}

Table IV shows that there is no monotonic relation between the number of subquestions and false-positive ratios. This finding can be explained as follows. When the total number of respondents was fixed, the number of subquestions increased and a higher number of respondents answered more than one subquestion incorrectly. Therefore, $N$ (not C) and $N$ (A and not C) increased. If we define the increased differences as $\Delta N$ (not $\mathrm{C}$ ) and $\Delta N$ (A and not $\mathrm{C}$ ), we can show with an easy calculation that if

$$
\frac{\Delta N(\mathrm{~A} \text { and } \operatorname{not} \mathrm{C})}{\Delta N(\operatorname{not} \mathrm{C})}>\frac{N(\mathrm{~A} \text { and not } \mathrm{C})}{N(\operatorname{not} \mathrm{C})}
$$

the false-positive ratio will increase. By contrast, if

$$
\frac{\Delta N(\mathrm{~A} \text { and } \operatorname{not} \mathrm{C})}{\Delta N(\operatorname{not} \mathrm{C})}<\frac{N(\mathrm{~A} \text { and } \operatorname{not} \mathrm{C})}{N(\operatorname{not} \mathrm{C})}
$$

the false-positive ratio will decrease. Since both cases occur equally, if the number of subquestions increases, the false-positive ratio will increase or decrease with an equal probability. Therefore, the number of subquestions is directly irrelevant to their validity.

\section{Contents of the subquestions}

Table IV also shows that although the value of $P_{2}$ (SQ.3) is smaller than the values of $P_{2}$ (SQ.1) and $P_{2}$ (SQ.2), its effect on the value of $P_{2}$ (SQ.1, SQ.2, and SQ.3) is negligible. This finding can be explained as follows. We found 97\% of respondents who answered SQ.3 incorrectly also answered SQ.1 and SQ.2 incorrectly. Therefore,

$N($ not $\mathrm{C}(\mathrm{SQ} .1, \mathrm{SQ} .2)) \simeq N($ not $\mathrm{C}(\mathrm{SQ} .1, \mathrm{SQ} .2$, and SQ.3) $)$,

and

$$
\begin{aligned}
& N(\mathrm{~A} \text { and not } \mathrm{C}(\mathrm{SQ} .1, \mathrm{SQ} .2)) \\
& \simeq N(\mathrm{~A} \text { and not } \mathrm{C}(\mathrm{SQ} .1, \mathrm{SQ} .2 \text {, and SQ.3) }) .
\end{aligned}
$$

Then, from Eq. (1), the effect of SQ.3 on $P_{2}$ (SQ.1, SQ.2, and SQ.3) is small. This analysis means that we could have omitted SQ.3, as things turned out.

The above process remains incomplete and it might become necessary to evaluate the validity of the subquestions from other points of view in the future. In other words, we need to carry out similar validation testing on the subquestions. However, considering the validity of the validation will only result in a series of discussion cycles. Therefore, we need to examine how and the extent to which the validity of the subquestions should be evaluated.

TABLE IV. The relation between the subquestions of Q.7 and the false-positive ratios. ${ }^{\text {a }}$

\begin{tabular}{llllllll}
\hline \hline & & & & & & & SQ.1 \\
Subquestions included in event C & & & SQ.1 & SQ.1 & SQ.2 & SQ.2 \\
\hline False-positive ratio of prelearners: $P_{1}(\mathrm{~A} \mid$ not C) & 0.41 & 0.34 & 0.35 & 0.40 & 0.39 & 0.38 & 0.39 \\
False-positive ratio of postlearners: $P_{2}(\mathrm{~A} \mid$ not C) & 0.71 & 0.71 & 0.54 & 0.72 & 0.69 & 0.67 & 0.71 \\
$Z$ score of the difference between $P_{1}$ and $P_{2}: Z_{12}$ & $2.78^{*}$ & $3.22^{*}$ & 1.47 & $3.09^{*}$ & $2.87^{*}$ & $2.71^{*}$ & $3.09^{*}$ \\
\hline \hline
\end{tabular}

${ }^{a}$ Each value is shown up to two decimal places. One star (*) represents there was a significant difference between $P_{1}$ and $P_{2}$ at the 0.05 level. 


\section{B. Validity of the classification of prelearners and postlearners}

We classified prelearners and postlearners according to their experience of learning the physics concept tested in a question. However, since the concept might be complex, we had to be careful not to extract one concept from several. Thus, we compared the false-positive ratios not only of physics concept prelearners and postlearners but also of mechanics prelearners and postlearners. We determined whether students had studied mechanics at high school from the survey. However, the difference in the classification was found to be small enough, and therefore the conclusion remained unchanged.

\section{Range of results}

Because our survey was limited to students at Meijo University, we would need to survey students from around the world in order to be able to generalize our conclusions. However, we expect that our results can be extrapolated because they are consistent with the results of the interviews with students at Tokyo Gakugei University, Nagoya University, and Meijo University. Further, Thornton et al. and Scott et al. found similar results for Q.16 [13,14].

Our survey only deals with the validity of Q.16 and Q.7. We should note, therefore, that even though these questions were proven to be invalid, this finding may not be true for the remaining FCI questions. Although we examined Q.16 and Q.7, as we found false positives for these questions in the interviews previously conducted, it is likely that the false-positive ratios of most other questions are lower. Hence, this study does not ignore other studies that have compared total FCI scores or evaluated FCI gain [21,22]. In the future, we might find the effect of the false positives on Q.16 and Q.7 relative to the total FCI score to be sufficiently small [23]. In that case, the FCI would be considered to have passed structural validation, and its validity would become more robust. In this study, we merely highlight the inadequacy of two FCI questions rather than evaluate the validity of the surveys that compare total FCI scores.

\section{SUMMARY}

In this study, we evaluated the structural validity of Q.16 and Q.7 in the FCI using subquestions. We statistically tested the difference in the false-positive ratios between prelearners and postlearners. For both Q.16 and Q.7, we found a significant difference between prelearners and postlearners at the 0.05 significance level. Based on this result, we determined that postlearners can answer both Q.16 and Q.7 without understanding the physics concept tested in the questions; therefore, the structures of these questions are invalid. Through this study, we highlighted the inadequacy of two FCI questions, although we did not assess the validity of surveys on FCI gain.

For future work, we plan to carry out the qualitative analysis of actual answer choices and patterns as well as quantitative analysis such as item response curves $[9,10]$. Moreover, we plan to disambiguate our data in order to assess the false-positive ratios by major area and analyze whether they differ among these populations.

If we were able to quantify the effect of the false positives on the total FCI score, we could regard this final number as part of the systematic error of the FCI. Similarly, if we evaluate and unify the systematic error from the validation of the distractor and the context [8-11], we could derive the systematic error of the FCI, thereby allowing us to compare the results considering these inadequacies without modifying them and to effectively measure the effect of physics education. This conclusion would enable our study to provide a positive and far-reaching effect in the field of physics education research.

The results of this study also have practical implications. Focusing on false positives allows for the development of a new diagnostic tool. Further, subquestions can be used to validate other diagnostic tools such as the force and motion concept evaluation [24]. By way of future research, researchers could reconstruct these subquestions as a diagnostic tool in order to measure the gradual understanding of physics concepts and integrate these questions into $e$-learning teaching material.

\section{ACKNOWLEDGMENTS}

We have greatly benefited from the discussion with K. Taniguchi (Kyoto University of Education) and M. Hull (Georgetown University). This work is supported in part by KAKENHI 24700875.
[1] D. Hestenes, M. Wells, and G. Swackhamer, Force concept inventory, Phys. Teach. 30, 141 (1992).

[2] I. Halloun and D. Hestenes, The initial knowledge state of college physics students, Am. J. Phys. 53, 1043 (1985); Common sense concepts about motion, 53, 1056 (1985).
[3] D. Huffman and P. Heller, What does the force concept inventory actually measure? Phys. Teach. 33, 138 (1995).

[4] P. Heller and D. Huffman, Interpreting the force concept inventory: A reply to Hestenes and Halloun, Phys. Teach. 33, 503 (1995). 
[5] E. Redish, Teaching Physics with the Physics Suite (Wiley, Hoboken, NJ, 2003), p. 96.

[6] L. Desimone and K. Le Floch, Are we asking the right questions? Using cognitive interviews to improve surveys in education research, Educ. Eval. Policy Anal. 26, 1 (2004).

[7] D. Hestenes and I. Halloun, Interpreting the force concept inventory: A response to March 1995 critique by Huffman and Heller, Phys. Teach. 33, 502 (1995).

[8] N. Rebello and D. Zollman, The effect of distracters on student performance on the force concept inventory, Am. J. Phys. 72, 116 (2004).

[9] G. A. Morris, L. Branum-Martin, N. Harshman, S. D. Baker, E. Mazur, S. Dutta, T. Mzoughi, and V. McCauley, Testing the test: Item response curves and test quality, Am. J. Phys. 74, 449 (2006).

[10] G. A. Morris, N. Harshman, L. Branum-Martin, E. Mazur, T. Mzoughi, and S.D. Baker, An item response curves analysis of the Force Concept Inventory, Am. J. Phys. 80, 825 (2012).

[11] J. Stewart, H. Griffin, and G. Stewart, Context sensitivity in the force concept inventory, Phys. Rev. ST Phys. Educ. Res. 3, 010102 (2007).

[12] J. Yasuda, H. Uematsu, and H. Nitta, Validating a Japanese version of force concept inventory, Phys. Educ. Soc. Jpn. (in Japanese) 59, 90 (2011) [http://ci.nii.ac.jp/naid/ $110008673371]$.

[13] R. K. Thornton, D. Kuhl, K. Cummings, and J. Marx, Comparing the force and motion conceptual evaluation and the force concept inventory, Phys. Rev. ST Phys. Educ. Res. 5, 010105 (2009).

[14] T.F. Scott, D. Schumayer, and A. R. Gray, Exploratory factor analysis of a Force Concept Inventory data set, Phys. Rev. ST Phys. Educ. Res. 8, 020105 (2012).

[15] These pictures are taken from E. Mazur, Peer Instruction (Prentice-Hall, Englewood Cliffs, NJ, 1996).

[16] M. Sato (unpublished) used a simpler method, wherein respondents are asked to write down the answer to an FCI question and explain the rationale behind their answer. The drawback of this method is that respondents do not always provide all the information necessary for researchers to examine whether they have an accurate understanding of the concept being tested by the question. Using subquestions allows us to overcome this uncertainty.

[17] In Japan, most high school students study two subjects from physics, chemistry, biology, and earth science. Most engineering students choose physics and chemistry, while most agriculture and humanities students choose chemistry and biology.

[18] D. Hestenes and J. Jackson, Revised Table II for the Force Concept Inventory, http://modeling.la.asu.edu/R\&E/ Research.html

[19] We designed the subquestions using the translation provided by M. Kurihara, K. Tsukamoto, H. Funahashi, A. Negishi, H. Nitta, H. Takahashi, and M. Kano (unpublished).

[20] It is common to use the 0.05 level of significance. See, R. A. Fisher, Statistical Methods for Research Workers (Oliver \& Boyd, London, 1925).

[21] R. Hake, Interactive-engagement versus traditional methods: A six-thousand-student survey of mechanics test data for introductory physics courses, Am. J. Phys. 66, 64 (1998).

[22] R. M. Goertzen, E. Brewe, L. H. Kramer, L. Wells, and D. Jones, Moving toward change: Institutionalizing reform through implementation of the learning assistant model and open source tutorials, Phys. Rev. ST Phys. Educ. Res. 7, 020105 (2011).

[23] The total FCI score is validated by analyzing its correlation with other diagnostic tools. For example, Thornton [13] and P. Nieminen, A. Savinainen, and J. Viiri, Relations between representational consistency, conceptual understanding of the force concept, and scientific reasoning, Phys. Rev. ST Phys. Educ. Res. 8, 010123 (2012).

[24] R. K. Thornton and D. R. Sokoloff, Assessing student learning of Newton's laws: The force and motion conceptual evaluation and the evaluation of active learning laboratory and lecture curricula, Am. J. Phys. 66, 338 (1998). 